REPORT 83-22
RER

Transport of water in frozen soil

I. Experimental determination of soil - water diffusivity under isothermal conditions

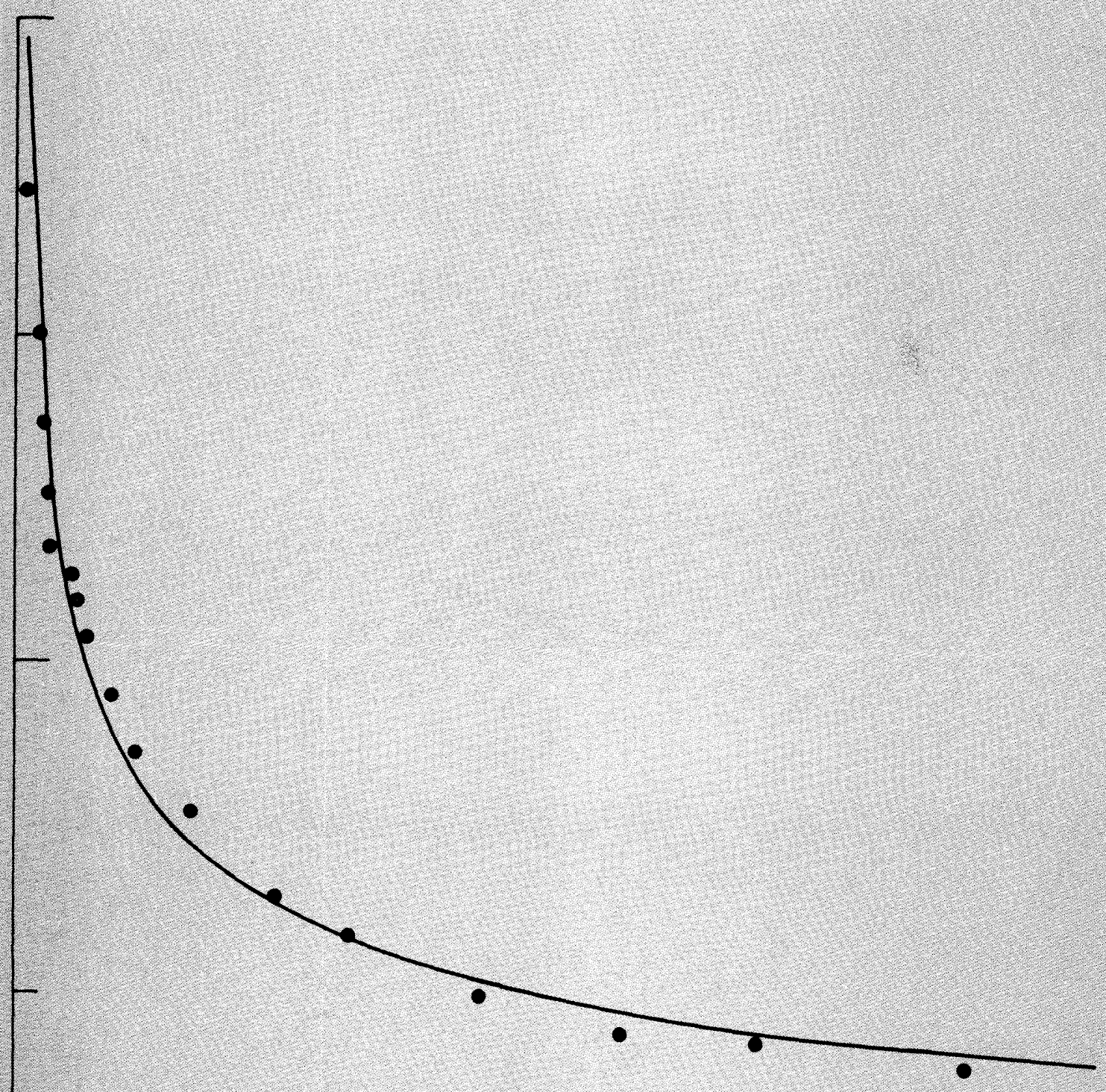




\section{CRREL Report 83-22}

August 1983

\section{Transport of water in frozen soil}

\section{Experimental determination of soil - water diffusivity under} isothermal conditions

Y. Nakano, A.R. Tice, J.L. Oliphant and T.F. Jenkins 
Unclassified

SECURITY CLASSIFICATION OF THIS PAGE (When Data Entered)

\begin{tabular}{|c|c|}
\hline REPORT DOCUMENTATION PAGE & $\begin{array}{l}\text { READ INSTRUCTIONS } \\
\text { BEFORE COMPLETING FORM }\end{array}$ \\
\hline \begin{tabular}{l|l|l} 
1. REPORT NUMBER & 2. GOVT ACCESSION NO. \\
CRREL Report $83-22$ &
\end{tabular} & 3. RECIPIENT'S CATALOG NUMBER \\
\hline $\begin{array}{l}\text { 4. TITLE (and Subtitle) } \\
\text { TRANSPORT OF WATER IN FROZEN SOIL } \\
\text { I. Experimental determination of soil-water diffusivity } \\
\text { under isothermal conditions }\end{array}$ & 6. PERFORMING ORG. REPORT NUMBER \\
\hline $\begin{array}{l}\text { 7. AUTHOR(s) } \\
\text { Y. Nakano, A.R. Tice, J.L. Oliphant and T.F. Jenkins }\end{array}$ & 8. CONTRACT OR GRANT NUMBER(s) \\
\hline $\begin{array}{l}\text { 9. PERFORMING ORGANIZATION NAME AND ADDRESS } \\
\text { U.S. Army Cold Regions Research and Engineering Laboratory } \\
\text { Hanover, New Hampshire } 03755\end{array}$ & $\begin{array}{l}\text { 10. PROGRAM ELEMENT, PROJECT, TASK } \\
\text { AREA \& WORK UNIT NUMBERS } \\
\text { ILIR 4A161101A91D }\end{array}$ \\
\hline \multirow{2}{*}{$\begin{array}{l}\text { 11. CONTROLLING OFFICE NAME AND ADDRESS } \\
\text { U.S. Army Cold Regions Research and Engineering Laboratory } \\
\text { Hanover, New Hampshire } 03755\end{array}$} & $\begin{array}{l}\text { 12. REPORT DATE } \\
\text { August } 1983\end{array}$ \\
\hline & $\begin{array}{l}\text { 13. NUMBER OF PAGES } \\
13\end{array}$ \\
\hline \multirow[t]{2}{*}{ 14. MONITORING AGENCY NAME \& ADDRESS(if different from Controlling Office) } & $\begin{array}{l}\text { 15. SECURITY CLASS. (of this report) } \\
\text { Unclassified }\end{array}$ \\
\hline & $\begin{array}{l}\text { 15a. DECLASSIFICATION/DOWNGRADING } \\
\text { SCHEDULE }\end{array}$ \\
\hline
\end{tabular}

16. DISTRIBUTION STATEMENT (of this Report)

Approved for public release; distribution unlimited.

17. DISTRIBUTION STATEMENT (of the abstract ontered in Block 20, if different from Report)

18. SUPPLEMENTARY NOTES

19. KEY WORDS (Continue on reverse side if necessary and ldentify by block number)

Experimental data

Frost heave

Frozen soil

Soil dynamics

Soil water

20. AESTRACT (Continue an reverab side if necesseany and idontify by block number)

A new experimental method for measuring the soil-water diffusivity of frozen soil under isothermal conditions is introduced. The theoretical justification of the method is presented and the feasibility of the method is demonstrated by experiments conducted using marine-deposited clay. The measured values of the soil-water diffusivity are found comparable to reported experimental data. 


\section{PREFACE}

This report was prepared by Y. Nakano, Chemical Engineer, Applied Research Branch, Experimental Engineering Division, A.R. Tice, Physical Science Technician, J.L. Oliphant, Research Physical Scientist, and T.F. Jenkins, Research Chemist, Earth Sciences Branch, Research Division, U.S. Army Cold Regions Research and Engineering Laboratory. Funding was provided by InHouse Laboratory Independent Research, ILIR 4A161101 A91D.

The contents of this report are not to be used for advertising or promotional purposes. Citation of brand names does not constitute an official endorsement or approval of the use of such commercial products. 


\title{
TRANSPORT OF WATER IN FROZEN SOIL I. Experimental determination of soil-water diffusivity under isothermal conditions
}

\author{
Y. Nakano, A.R. Tice, J.L. Oliphant and T.F. Jenkins
}

\section{INTRODUCTION}

It is known (Anderson et al. 1978) that water in frozen soil generally exists in three phases: vapor, liquid water and ice. The liquid-phase water in frozen soil, which is believed to be mobile, is often referred to as unfrozen water (Anderson et al. 1978). The unfrozen water content depends on the soil type and temperature, and monotonically decreases with decreasing temperature. It is known that some clay contains up to $5 \%$ unfrozen water by weight at $-10^{\circ} \mathrm{C}$. Although it has been widely assumed that the unfrozen water content is independent of the ice content, recent work (Tice et al. 1978) using the nuclear magnetic resonance technique indicates that unfrozen water noticeably increases with increasing ice content.

It has been recognized that accurate knowledge of water movement in frozen soils is very important for many practical applications. For example, frost heave is known to be caused by transport of water through freezing soils (Loch and Miller 1975) and the damage of frost heave is of major economic concern to both agriculturalists and engineers.

Williams and Burt (1974) measured the hydraulic conductivity of frozen soil using a permeameter in which frozen soil was positioned between two compartments filled with an aqueous solution of lactose. However, the presence of significant concentrations of lactose inevitably introduces a deviation in the system to some unknown degree. In order to overcome this difficulty Horiguchi and Miller (1980) introduced a different kind of permeameter, in which frozen soil was positioned between two compartments filled with pure supercooled water, and measured the hydraulic conductivity of frozen soil in the temperature range of $0^{\circ}$ to $-0.15^{\circ} \mathrm{C}$. The lowest temperature attainable in this permeameter is evidently restricted by freezeup of the supercooled water.

In the two methods of permeability measurement described above, the lowest attainable temperature is restricted one way or another. In this paper, we will introduce a new and simple method that could be used to measure the rate of water transport in frozen soil at any temperature. We will present the theoretical justification of this new method and some experimental data to demonstrate the feasibility of the method.

When we consider water transport in frozen porous media under isothermal conditions, it is reasonable to expect that this problem can be treated in a manner similar to the problem of unfrozen soil under the condition that either ice is absent or the effects of ice on water transport are negligible. Under such conditions water transport in frozen soil corresponds to that in unfrozen soil in which the volumetric water content is less than $10 \%$ or so.

Historically in soil physics, the problem of water transport in soil containing a small amount of water has been subjected to intensive investigations (Swartzendruber 1969). If we assume that the medium obeys the extended Darcy's law for unsaturated porous flow and that gravitational effects are negligible, the flux of water $F$ in one direction under an isothermal condition is given as

$$
F=-D(\theta) \rho \frac{\partial \theta}{\partial x}
$$


where $\theta$ is the weight of water per weight of dry soil, $x$ is the space coordinate, $\rho$ is the dry density and $D(\theta)$ is the soil-water diffusivity (we will use the water content based upon weight throughout this work). When the transport of water in the vapor phase is included, $D(\theta)$ is given (Philip 1957)

$$
D(\theta)=\sum_{\mathrm{i}=1}^{3} D_{\mathrm{i}}(\theta)
$$

where $D_{1}, D_{2}$ and $D_{3}$ are the diffusivities of absorbed liquid water, vapor, and pore liquid water, respectively. Raats (1975) has investigated eq 2 by using the mixture theory of continuum mechanics and has shown the validity of eq 2 if the motion of the gaseous phase in the system does not significantly affect the transport of water.

In frozen soil almost all pore liquid water is believed to be frozen (Anderson et al. 1978) and we have

$$
D(\theta)=D_{1}(\theta)+D_{2}(\theta) .
$$

It is anticipated that the transport of water in frozen soil occurs in two phases, absorbed liquid water and vapor, and that an overall diffusivity $D(\theta)$ is given by the simple sum of the diffusivities of both phases.

When eq 1 holds true, the equation of continuity is given as

$$
\frac{\partial \theta}{\partial t}=\frac{\partial}{\partial x}\left[D(\theta) \frac{\partial \theta}{\partial x}\right]
$$

where $t$ is time. The commonly used method for the experimental measurement of $D$ utilizes the mixed initial and boundary value problem of eq 4 with initial and boundary conditions given as

$$
\begin{aligned}
\theta(x, 0) & =\theta_{1} & & x=0 \\
& =\theta_{2} & & 0<x \\
\theta(0, t) & =\theta_{1} & & 0 \leqslant t
\end{aligned}
$$

where $\theta_{1}$ and $\theta_{2}$ are constants with $\theta_{1}>\theta_{2}$. In the reported experiments the water content $\theta_{1}$ is kept constant by either a fritted glass bead plate (Nielson et al. 1962) or a diffusion chamber (Jackson 1965).

In this work we will introduce the method of experimental measurement by using the initial value problem of eq 4 in order to avoid the inconvenience of maintaining the water content constant at the boundary.

\section{THEORY}

Our experiment consists of two long columns of soil with the same size and dry density. One of them is uniformly dry with the negligibly small water content $\theta=0$ while the other is uniformly wet with $\theta=\theta_{0}>0$. The wet column is allowed to contain ice only for the case, if it exists, in which the effects of ice on water transport are negligible. At time $t=0$ we connect the two columns to make a single column from which no water escapes. While we maintain the column at the specified constant temperature, water is transported from the wet part to the dry part. This experiment can be described in mathematical terms by the following initial value problem of eq 4 on the set, $S=\{(-\infty, \infty) x(0, T)$; $T>0\}$ :

$$
\begin{array}{cc}
\frac{\partial \theta}{\partial t}=\frac{\partial}{\partial x}[D(\theta) & \left.\frac{\partial \theta}{\partial x}\right] \text { on } S \\
\theta(x, 0)=\theta_{0} & x<0 \\
=0 & x \geqslant 0 .
\end{array}
$$

Oleinik et al. (1958) investigated the general initial value problem of eq $6 a$ and have shown the existence and uniqueness of the solution under the conditions that $\theta(x, 0)$ satisfies the Lipschitz condition for $-\infty<x<\infty$, that $D(\theta)$ has continuous derivatives of the fourth order, and that $D(\theta)$ is bounded for bounded $\theta$. It should be mentioned that the solution defined (Oleinik et al. 1958) may not be continuously differentiable and is often referred to as a weak (generalized) solution. Unfortunately, the existence and uniqueness theorem (Oleinik et al. 1958) is not directly applicable to the problem of eq $6 a$ and $b$ because the initial condition of eq $6 b$ obviously does not satisfy the Lipschitz condition.

Recently, Nakano (in press) investigated a special case of the problem of eq $6 \mathrm{a}$ and $\mathrm{b}$ in which $D(\theta)$ is given as

$$
D(\theta)=D_{0}=D_{0} \theta^{\beta}
$$

where $D_{0}$ and $\beta$ are positive numbers. Equation 6a can be transformed into an ordinary differential equation by introducing the similarity variable $\eta=x\left[D_{0}\right.$ $\left.\left(t+t_{0}\right)\right]^{-1 / 2}$ where $t_{0} \geqslant 0$ is a constant. The similarity solution $\theta(x, t)=f(\eta)$ should satisfy the equation

$$
\left[f^{\beta} f^{\prime}\right]^{\prime}+(1 / 2) \eta f^{\prime}=0 \quad-\infty<\eta<\infty
$$


where primes denote differentiation with respect to $\eta$. The solution $f(\eta)$ is required to satisfy the conditions

$$
f(-\infty)=\theta_{0} \quad f(\infty)=0
$$

Nakano (in press) has shown that there exists at least one continuous solution of eq $8 \mathrm{a}$ and $\mathrm{b}$. This solution is strictly monotone-decreasing and has the property given as

$$
\begin{array}{ll}
f(\eta)>0 & \text { for } \sigma>\eta \\
f(\eta)=0 & \text { for } \sigma \leqslant \eta
\end{array}
$$

where $\sigma$ is a positive number. We will call the interface between the part where $\theta>0$ and the part where $\theta=0$ a wetting front. It follows from eq 9 that the location of the wetting front $X(t)$ is given as

$$
X(t)=\sigma D_{0}^{1 / 2}\left(t+t_{0}\right)^{1 / 2}
$$

For convenience's sake we define the functions, $f_{1}(\eta)$ and $f_{2}(\eta)$, as

$$
\begin{array}{llll}
f_{1}(\eta) \equiv f(\eta) & \text { if } & t_{0}=0 \\
f_{2}(\eta) \equiv f(\eta) & \text { if } & t_{0}>0 .
\end{array}
$$

It has been shown (Nakano, in press) that the solution $f_{1}(\eta)$ is not unique and contains one unspecified parameter. Choosing $\sigma$ to be this unspecified parameter, we will write $f_{1}(\eta)$ as $f_{1}(\eta ; \dot{\sigma})$. This one-parameter family of solutions $f_{1}(\eta ; \sigma)$ satisfies the initial condition equation (eq $6 \mathrm{~b}$ ) and also provides solutions to the problem of eq $6 a$ and $b$, implying that the problem of eq $6 a$ and $b$ has many solutions. The solution $f_{2}(\eta)$ does not satisfy the initial condition equation (eq 6b). Instead, the initial data of $f_{2}(\eta)$ have the property given as

$$
\begin{array}{ll}
f_{2}(\eta)>0 & \eta \leqslant \sigma t_{0}^{1 / 2} \\
f_{2}(\eta)=0 & \eta \geqslant \sigma t_{0}^{1 / 2} .
\end{array}
$$

Unlike $f_{1}(\eta ; \sigma), f_{2}(\eta)$ is shown to be unique.

Studying the asymptotic behavior of the solution to the wide class of initial value problems of eq $6 \mathrm{a}$, Nakano (in press) has shown that the solutions of eq $6 \mathrm{a}$ and $\mathrm{b}, f_{1}(\eta ; \sigma)$, asymptotically converge upon $f_{2}(\eta)$ as time tends to infinity with the proper choice of $\sigma$. The proper value of $\sigma$ cannot be determined from the initial condition equation (eq 6b), but from the experimental data as described below.

The properties of the solution of eq $8 \mathrm{a}$ and $\mathrm{b}$, $f(\eta)$, were investigated (Nakano, in press) and it has been shown that $f_{2}(\eta)$ is not continuously differentiable because the transition between the part $f>0$ and the part $f=0$ is not smooth. In left neighborhoods of the wetting front $(\sigma-\epsilon<\eta<\sigma ; \epsilon>0) f(\eta)$ is found to behave as

$$
f(\eta)=(\sigma \beta / 2)^{1 / \beta}(\sigma-\eta)^{1 / \beta}
$$

The derivatives of $f(\eta)$ are given as

$$
\begin{aligned}
& f^{\prime}(\eta)=-\beta^{-1}(\sigma \beta / 2)^{1 / \beta}(\sigma-\eta)^{(1-\beta) / \beta} \\
& f^{\prime \prime}(\eta)=(1-\beta) \beta^{-2}(\sigma \beta / 2)^{1 / \beta}(\sigma-\eta)^{(1-2 \beta) / \beta}
\end{aligned}
$$

$$
f^{\prime \prime \prime}(\eta)=-(1-\beta)(1-2 \beta) \beta^{-3}(\sigma \beta / 2)^{1 / \beta}(\sigma-\eta)^{(1-3 \beta) / \beta}
$$

etc. From the above equations we find the following. If $\beta>1, f^{\prime}(\eta)$ tends to minus infinity as $\eta$ tends to $\sigma$ and $f^{\prime \prime}(\eta)<0$. This implies that the wetting front is very steep and convex upwards. This type of wetting front was reported (Nielson et al. 1962). If $1 \geqslant \beta>0$, $f^{\prime}(\eta)$ remains finite and $f^{\prime \prime}(\eta)>0$. This type of wetting front was observed (Jackson 1964). It is clear that the transition between the part $f>0$ and the part $f=0$ becomes smoother as $\beta$ decreases. When $\beta=0$, it is known that there is a continuously differentiable solution given as

$$
\theta(\eta)=\left(\theta_{0} / 2\right)[1-\operatorname{erf}(\eta / 2)]
$$

where erf is the error function defined as

$$
\operatorname{erf}(x)=\frac{2}{\sqrt{\pi}} \int_{0}^{x} \exp \left(-s^{2}\right) d s
$$

It follows from eq 15 that $\theta$ tends to zero as $\eta$ approaches plus infinity. Hence, according to our definition, there appears no wetting front in this solution. Equation 15 may be written as

$$
\theta_{0}-\theta(\eta)=\left(\theta_{0} / 2\right) \operatorname{erfc}(-\eta / 2)
$$

where erfc is the complemental error function defined as 


$$
\operatorname{erfc}(x)=1-\operatorname{erf}(x)
$$

When $\eta$ approaches minus infinity, the solution $f(\eta)$ has been shown (Nakano, in press) to behave as

$$
\theta_{0}-f(\eta)=\left(\theta_{0} / 2\right) \operatorname{erfc}\left[-\eta /\left(2 \theta_{0}^{\beta / 2}\right)\right]
$$

The behavior of $f(\eta)$ resembles $\theta(\eta)$ near $\eta=-\infty$.

When $D(\theta)$ is given as in eq 7 , we would measure the profile of water content with time in our experiment. It is expected that after some time $t=T$, the profile will become stable and vary little with time afterward. We take the profile at $t=T$ as an experimental asymptotic curve $f^{*}(\eta)$. By comparing $f^{*}(\eta)$ with the theore tical profile $f_{1}(\eta ; \sigma)$ we can, in principle, determine the values of $\sigma, D_{0}$ and $\beta$. For instance, from the measured locations of a wetting front, $X(T)$, we find

$$
\sigma D_{0}^{1 / 2}=X(T) T^{-1 / 2} \text {. }
$$

In the next step we seek approximate values of $D_{0}$ and $\beta$ using eq 13 . Equation 13 may be written as

$$
Z \equiv \ln f=\alpha+\beta^{-1} Y
$$

where

$$
\begin{aligned}
& \alpha=\beta^{-1} \ln \left(\sigma \beta D_{0}^{-1 / 2} / 2\right) \\
& Y=\sigma D_{0}^{1 / 2}-x T^{-1 / 2} .
\end{aligned}
$$

Using several experimental points $(x, f)$ near the wetting front, we plot $Z$ vs $Y$. The graph $(Y, Z)$ is expected to be approximately linear. Thus we can determine the approximate values of $D_{0}$ and $\beta$.

In the final step we seek the values of $D_{0}$ and $\beta$ that minimize the difference between $f^{*}(\eta)$ and $f_{1}(\eta ; \sigma)$ by trial and error. The theoretical profile $f_{1}(\eta ; \sigma)$ can be obtained by the numerical integration of eq $8 \mathrm{a}$. We will describe below one of several possible methods of integration that we use in this work.

For convenience's sake we introduce two new variables defined as

$$
\begin{aligned}
& s=\eta / \sigma \\
& y=f / \sigma^{2 / \beta} .
\end{aligned}
$$

Using eq $23 a$ and $b$, we reduce eq $8 a, 13$ and $14 a$ to

$$
\left(y^{\beta} y^{\prime}\right)^{\prime}+1 / 2 s y^{\prime}=0 \quad-\infty<s \leqslant 1
$$

$$
\begin{aligned}
& y=(\beta / 2)^{1 / \beta}(1-s)^{1 / \beta} \\
& y^{\prime}=-\beta^{-1}(\beta / 2)^{1 / \beta}(1-s)^{(1-\beta) / \beta}
\end{aligned}
$$

where primes denote differentiation with respect to $s$. In order to reduce eq 24 to a system of firstorder equations, we introduce another dependent variable defined as

$$
z=-y^{\beta} y^{\prime}
$$

Now, eq 24 is reduced to

$$
\begin{aligned}
& y^{\prime}=-y^{-\beta} z \equiv F(s, y, z) \\
& z^{\prime}=-1 / 2 s y^{-\beta} z \equiv G(s, y, z)
\end{aligned}
$$

with conditions

$$
y(1)=z(1)=0
$$

We will simultaneously integrate eq $27 \mathrm{a}$ and $\mathrm{b}$ by the Runge-Kutta method from $s=1$ to any desired value of $s<1$ using eq 25 and 26 for evaluating $F$ and $G$ at the first step of integration. Since $y$ varies most rapidly near the wetting front $s=1$ and the rate of change decreases with decreasing $s$, it is desirable to use variable increments. Particularly, when $\beta$ is greater than 1 , the selection of the first step increment should be carefully examined in order to attain the required accuracy of computation. In the present work the value of $\beta$ is much less than 1 and we computed the profile of water content with a relative error of less than $1 \%$ using the initial increment of $10^{-5}$.

\section{EXPERIMENT}

The soil selected for this study, called Morin clay, is a marine-deposited clay obtained from the Morin brickyard, Auburn, Maine. This area was uplifted and the original solutes leached by natural seepage. The specific surface area is $60 \mathrm{~m}^{2} / \mathrm{g}$ soil, which may be considered typical for clay of this texture. We present the measured unfrozen water content in Morin clay with $21 \%$ total water content at various temperatures together with the fitted curve in Figure 1. Figure 1 shows that about $10 \%$ of the water remains unfrozen at $-1.0^{\circ} \mathrm{C}$, the temperature selected for this study.

We used $10-\mathrm{mL}$ graduated plastic pipettes to enclose the soil columns. The dispensing end of each 


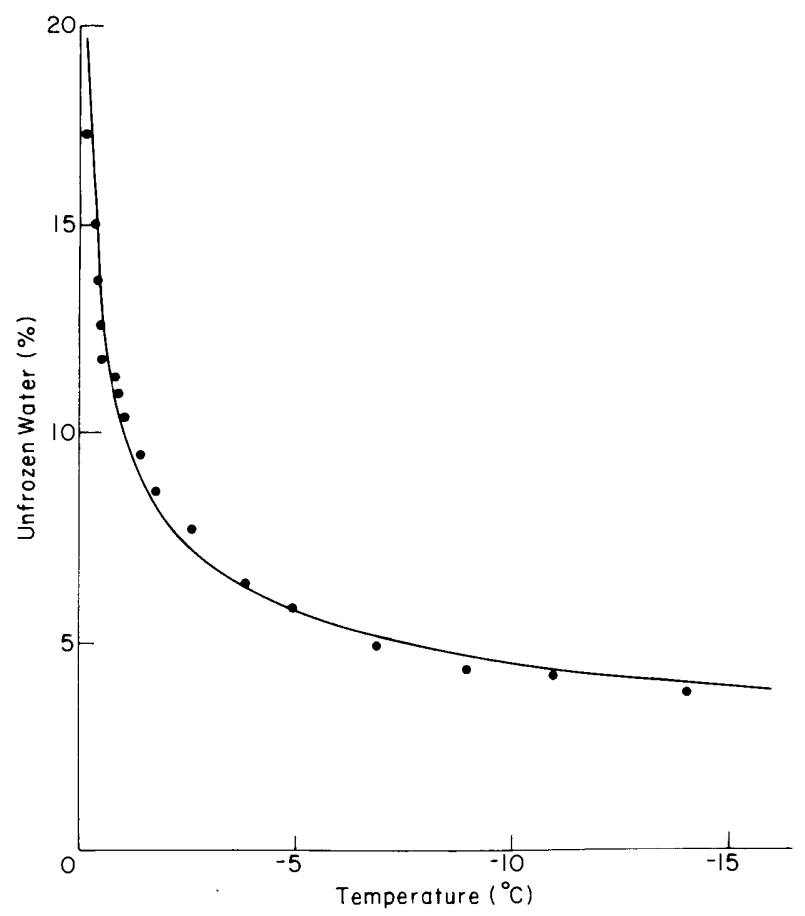

Figure 1. Unfrozen water content of Morin clay with $21 \%$ total water content versus temperature.

pipette was cut off and sealed by a stopper. The dimensions of each soil column were $0.80 \mathrm{~cm}$ in diameter by $20.33 \mathrm{~cm}$ in length. Each experiment of this study required the preparation of two soil columns with the same specified soil dry density. One of them was uniformly dry with a negligibly small water content while the other was uniformly wet.

We chose the average soil dry density to be 1.35 $\mathrm{g} / \mathrm{cm}^{3}$ and the water content of the wet column to be $8.00 \%$ in this investigation. Since all experiments were conducted under the isothermal condition of $-1.0^{\circ} \mathrm{C}$, it is clear from Figure 1 that no ice was present in this study. For the preparation of wet columns, we added $16 \mathrm{~g}$ of distilled water to $200 \mathrm{~g}$ of oven-dry Morin clay. Mixing thoroughly the water and soil, we allowed the mixture to set for at least 3 days to attain moisture equilibration.

The uniform packing of soil into a pipette is one of the most critical operations in this experimental work. Despite frequent weighing of the soil-filled pipettes, we had to discard some of them due to lack of uniformity in terms of dry density. For the preparation of dry columns, oven-dry Morin clay was packed into a pipette with slightly greater compactive force. During the packing, Morin clay gained a small amount of water from the air, and the water content of dry columns averaged about $0.4 \%$ at the end of packing. Immediately after the packing was finished, we sealed the open end with a sleave type of rubber stopper and allowed the sample to set for at least 3 days for moisture reequilibration. Wet columns were frozen down to $-16.0^{\circ} \mathrm{C}$ and gradually warmed up to $-1.00^{\circ} \mathrm{C}$.

An experiment began by joining two pipettes, one with dry soil and one with wet soil, which were both set at $-1.0^{\circ} \mathrm{C}$. Upon removing the sleave-type stoppers from these two pipettes, we joined them with surgical rubber tubing. Both surfaces of contact were made smooth in order to avoid contact resistance. Immediately after the two pipettes were joined, they were placed in a constant temperature

Table 1. Mean $\left(\mathrm{g} / \mathrm{cm}^{3}\right)$ and standard deviation of dry density.

\begin{tabular}{cccc} 
Exp. series & $\begin{array}{c}\text { Time duration } \\
\text { (day) }\end{array}$ & Mean & $\begin{array}{c}\text { Standard } \\
\text { deviation }\end{array}$ \\
\hline \multirow{2}{*}{1} & 2 & 1.35 & 0.102 \\
& 4 & 1.36 & 0.120 \\
& 8 & 1.35 & 0.111 \\
& 16 & 1.35 & 0.131 \\
2 & & & \\
& 4 & 1.36 & 0.122 \\
& 8 & 1.34 & 0.123 \\
& 16 & 1.35 & 0.116 \\
\hline
\end{tabular}


bath containing an ethylene glycol-water mixture. The bath temperature was set at $-1.0^{\circ} \mathrm{C}$ and was maintained to within $\pm 0.03^{\circ} \mathrm{C}$ using a Bayley proportional temperature controller.

After a specified time passed, the jointed pipette was removed from the constant temperature bath and quickly sectioned into a total of 64 equal thin column segments. Each segment was placed in a glass weighing bottle and oven-dried overnight. The water content and the dry density of each segment were determined gravimetrically.

The time durations were $2,4,8$ and 16 days for the first series of experiments. In order to examine the reproducibility of the experiments, we conducted the second series of experiments where the experimental times were set $4,8,16$ and 32 days.

The uniformity of the soil columns was examined by using the measured dry density of segments after each experiment. The mean and the standard deviation of dry density calculated are given in Table 1 .

\section{RESULTS AND DISCUSSION}

We present the experimental results in Figure 2 where the water content $\theta$ is plotted versus the distance $x$. As described above we have two sets of data at $t=4,8$ and 16 days. These two sets of profiles in Figure 2 indicate that it is difficult to accurately reproduce the same profile, and that the difference between the two profiles amounts to about 0.5 in terms of percentage of water content, which corresponds to about $10 \%$ relative error in the wetter end of a soil column.

According to the results of the mathematical analysis described in the previous section, experimental profiles are expected to converge upon an asymptotic profile as time increases if $D(\theta)$ is given by eq 7 . In order to examine the asymptotic behavior of experimental profiles, in Figure 3 we plotted the water content $\theta$ versus $\xi$ given as

$$
\xi=D_{0}^{1 / 2} \eta=x t^{-1 / 2}
$$

In Figure 3 we used the average values of two sets of data at $t=4,8$ and 16 days. This figure indicates that the experimental profiles coincide with each other reasonably well after 8 days. We conclude that experimental profiles tend to stabilize as if they were converging upon an asymptotic profile as time increases.

In the next step we will examine how well eq 7 expresses the actual behavior of $D(\theta)$ of Morin clay. Since the initial water content of dry columns of

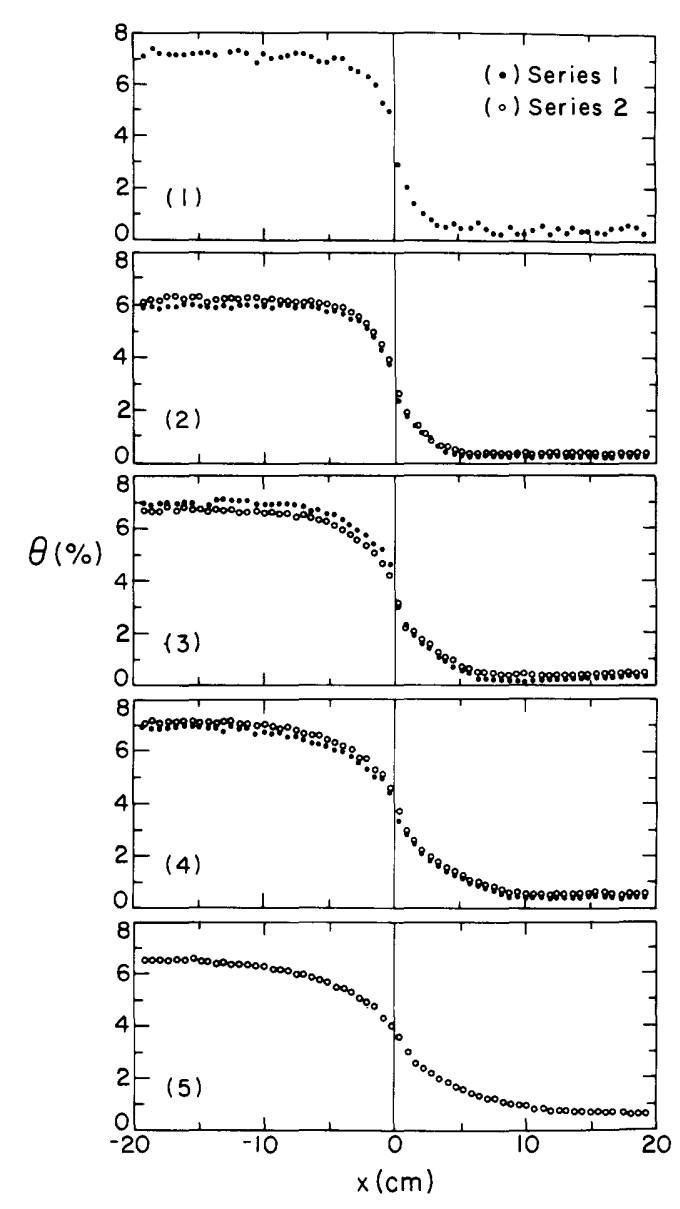

Figure 2. Water content profiles: $(1) \mathrm{t}=2$ days, (2) $\mathrm{t}=4$ days, (3) $\mathrm{t}=8$ days, (4) $\mathrm{t}=16$ days, $(5) \mathrm{t}=32$ days.

soil is not negligibly small in our experiments, we introduce a new variable $u$ given as

$$
u=\theta-\theta_{\mathrm{c}}
$$

where $\theta_{c}$ is the initial water content of dry columns. Using eq 30 we reduce eq 7 to

$$
D(\theta)=D_{0}\left(\theta-\theta_{c}\right)^{\beta} .
$$

It is easy to find that the results of the mathematical analysis described in the previous section hold true in terms of the new variable $u$ if $D(\theta)$ is given by eq 31 .

Using the least-squares method we determined the parameters in eq 31 for the averaged experimental profiles at $t=8$ days as

$$
D(\theta)=1.21\left(\theta-3.8 \times 10^{-3}\right)^{0.272}
$$

where $D(\theta)$ is in $\mathrm{cm}^{2} /$ day and $\theta$ is in g water/g dry soil. The curve in Figure 3 is the computed profile 


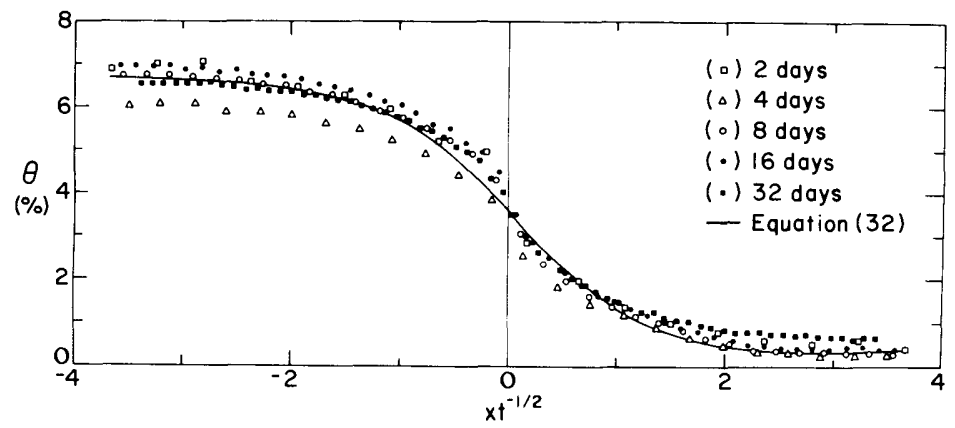

Figure 3. Water content versus $\xi$.

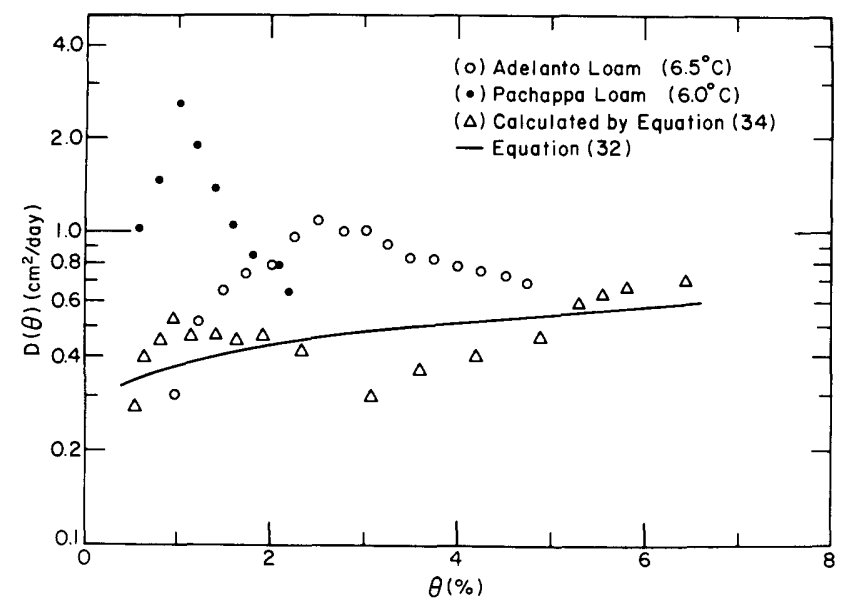

Figure 4. Soil-water diffusivity versus water content (Adelanto and Pachappa loams from Jackson [1965] ).

using eq 32 and the Runge-Kutta method described earlier. The computed profile appears to be a reasonably good approximation, but is not quite a close fit for the experimental profiles.

In order to examine the reason why eq 31 does not provide a close fit, we will evaluate $D(\theta)$ directly from the experimental profile at $t=8$ days under an assumption described below. Using the similarity variable $\xi$, we reduce eq $6 a$ and $b$ to

$$
\begin{aligned}
& {\left[D(f) f^{\prime}\right]^{\prime}+1 / 2 \xi f^{\prime}=0 \quad-\infty<\xi<\infty} \\
& f(-\infty)=\theta_{0} \quad f(\infty)=0 .
\end{aligned}
$$

We assume that the solution of eq $6 a$ and $b$ asymptotically converges upon that of eq $33 \mathrm{a}$ and $\mathrm{b}$ regardless of the functional form of $D(\theta)$ if $D(\theta) \geqslant 0$ is a smooth function of $\theta$. When this assumption holds true, by integrating eq 33 a we obtain

$$
D[\theta(\xi)]=\left[\theta_{c} \xi_{0}-\xi \theta+\int_{\xi_{0}}^{\xi} \theta d \xi\right] /\left[2 \frac{d \theta}{d \xi}\right]
$$

where $\xi_{0}$ is the value of $\xi$ at the wetting front. Using the experimental profile at $t=8$ days, we have approximately evaluated the right-hand side of eq 34 to obtain $D(\theta)$. We have plotted the calculated values of $D(\theta)$ versus $\theta$ in Figure 4 where the curve is eq 32. In spite of inherent inaccuracies in evaluating derivatives from experimental data, it appears that there is a slight peak in $D(\theta)$ near $\theta=1 \%$. Due to uncertainty as to the validity of this result, we can only speculate that eq 31 does not provide a close fit for experimental profiles because the actual $D(\theta)$ may not be a monotonically increasing function of $\theta$. 
It seems that no one has reported the soil-water diffusivity of frozen soils. Jackson (1965) measured the diffusivity of Adelanto loam and Pachappa loam at temperatures of $6.5^{\circ} \mathrm{C}$ and $6.0^{\circ} \mathrm{C}$, respectively. We plotted Jackson's data in Figure 4. It is known that $D(\theta)$ decreases as temperature decreases. Assuming that Jackson's theoretical prediction based upon the concept of activation energy can be extended to $-10^{\circ} \mathrm{C}$, we roughly estimate that the diffusivity at $-1.0^{\circ} \mathrm{C}$ would be three-quarters to one-half of that at $6.0^{\circ} \mathrm{C}$. This brings Jackson's and the present data closer together, and our measured values of the soil-water diffusivity are comparable to Jackson's data.

\section{CONCLUDING REMARKS}

We have introduced a new experimental method for measuring the soil-water diffusivity of frozen soil under isothermal conditions. We have presented the theoretical justification of the method and demonstrated the feasibility of the method with an experiment using Morin clay.

The theoretical justification is made under the assumption that the soil-water diffusivity is proportional to some power of water content. We have found that this functional form of the soil-water diffusivity does not provide a close fit for the experimental data obtained. Thus we should consider the measured diffusivity approximate. The approximation was needed in analyzing experimental data because the mathematical theorems currently available are limited. Mathematical theorems for the more general case of a diffusivity function are required to improve the accuracy in data analysis.

\section{LITERATURE CITED}

Anderson, D.M., R. Pusch and E. Penner (1978) Physical and thermal properties of frozen ground. Geotechnical Engineering for Cold Regions (O.B. Andersland and D.M. Anderson, Eds.). New York: McGraw-Hill, pp 37.

Horiguchi, K. and R.D. Miller (1980) Experimental studies with frozen soil in an 'ice sandwich' permeameter. Cold Regions Science and Technology, 3: 177 .

Jackson, R.D. (1964) Water vapor diffusion in relatively dry soil: I. Theoretical considerations and sorption experiments. Soil Sciences Society of American Proceedings, 28: 172.

Jackson, R.D. (1965) Water vapor diffusion in relatively dry soil: IV. Temperature and pressure effects on sorption diffusion coefficients. Soils Science Society of American Proceedings, 29: 144.

Loch, J.P.G. and R.D. Miller (1975) Tests of the concept of secondary frost heaving. Soils Science Society of American Proceedings, 39: 1036.

Nakano, Y. (in press) Asymptotic behavior of solutions of the problem of wetting fronts in one-dimensional, horizontal and infinite porous media. $A d$ vances in Water Resources.

Nielson, D.R., J.W. Biggar and J.M. Davidson (1962)

Experimental considerations of diffusion analysis in unsaturated flow problems. Soils Science Society of American Proceedings, 26: 107.

Oleinik, O.A., A.S. Kalashnikov and C. Yui-Lin (1958) The Cauchy problem and boundary problems for equations of the type of non-stationary infiltration. Izv. Akad. Nauk. USSR Ser. Mat, 22: 667.

Philip, J.R. (1957) The theory of infiltration: 1. The infiltration equation and its solution. Soil Sciences, 83: 345.

Raats, P.A.C. (1975) Transformations of fluxes and forces describing the simultaneous transport of water and heat in unsaturated porous media. Water Resources Research, 11: 938

Swartzendruber, D. (1969) The flow of water in unsaturated soils. In Flow Through Porous Media (J.M. DeWiest, Ed.). New York: Academic Press, pp. 215.

Tice, A.R., C.M. Burrows and D.M. Anderson (1978) Phase composition measurements on soils at very high water contents by the pulsed nuclear magnetic resonance technique. Transportation Research Record, No. 675, pp. 11.

Williams, P.J. and T.P. Burt (1964) Measurement of hydraulic conductivity of frozen soils. Canadian Geotechnical Journal, 11 : 647. 